\title{
Associations and hallucinations in mice and men
}

\author{
Dominic M. Dwyer ${ }^{1}$ \\ Published online: 5 February 2018 \\ (C) Psychonomic Society, Inc. 2018
}

\section{Summary}

Powers et al. (2017, Science, 357(6351), 596-600) report that Pavlovian conditioning can result in the perception of a stimulus in its absence, and that this effect is related to hallucinations outside the laboratory. Considered alongside similar studies in animals, this suggests that associatively produced perceptual processing offers a means to study hallucination-like behaviour in the animal laboratory.

Keywords Mediated conditioning $\cdot$ Sensory preconditioning $\cdot$ Psychosis $\cdot$ Schizophrenia $\cdot$ Animal models

One of the most well-known associative learning effects is Pavlovian conditioning: pairing a neutral stimulus (CS; conditioned stimulus) with a motivationally relevant stimulus (US; unconditioned stimulus) results in the CS alone subsequently eliciting conditioned responses (CR). Despite is ubiquity, this superficially simple procedure can be surprising and highly informative. While hallucinations (sensory perceptions without relevant external experience) may seem a matter far removed from the behavioural laboratory, an intriguing recent study of associatively induced hallucinations by Powers, Mathys, and Corlett (2017) suggests otherwise.

The experiment used a $2 \times 2$ design, involving participants diagnosed with psychotic illness plus controls with no diagnosis, and within each group participants who reported hearing voices and those who did not. The behavioural task was also simpledetection thresholds for tones embedded in white noise were established at $75 \%, 50 \%$, and $25 \%$ likelihood levels, and a checkerboard stimulus established as a cue for tone presentation. Across testing, detection of the tone was examined when it was presented at these levels and on trials where the checkerboard cue was presented without tone stimulation. On these critical trials, "detection" of the tone in the presence of a previously associated visual cue is functionally equivalent to hallucination: Auditory stimuli are perceived despite not being present. Although present to some degree in all participants, this occurred more frequently in participants who reported hearing voices outside the laboratory. Moreover, fMRI analysis suggested that activity when the tone was reported on visual-only trials

Dominic M. Dwyer

DwyerDM@cardiff.ac.uk

1 School of Psychology, Cardiff University, Tower Building, Park Place, Cardiff CF10 3AT, UK overlapped with tone-responsive brain regions, supporting the idea that the tone was actually perceived despite its absence. Activity on these trials also overlapped with a network of brain regions previously implicated in hallucinations. Additional analysis of the behavioural data using a hierarchical Bayesian model, with a critical parameter being the weighting of prior beliefs relative to current sensory input when determining a response, suggested that voice-hearing participants (independent of psychosis) gave proportionally greater weighting to prior beliefs.

Of course, critical comments are possible. Using a single CS leaves open the question of whether the effect was due to true conditioning or pseudoconditioning (responses elicited following presentation of a US that are not dependant on a CS-US association). Some hint is given by a signal detection analysis suggesting that voice-hearing participants had more liberal response criteria. The study also makes much of correlations between the propensity to "detect" the tone on tone-absent trials and measures of hallucination severity, but as correlations were taken across the whole sample, they confound group differences with individual variation and are thus uninformative. Notwithstanding these issues, Powers et al. (2017) have provided a clear demonstration that Pavlovian conditioning, where a visual cue was paired with an auditory cue, can result in perception of the auditory stimulus after presentation of the visual cue alone. This associatively based hallucination effect was more pronounced in individuals who reported hallucinations and hearing voices in daily life.

Powers et al. (2017) discuss the implications of their results for the pathology and treatment of hallucinations, but there is also a broader context because the results are strikingly similar to prior animal research in the laboratory. For example, odour cues previously paired with sucrose are able to activate the same gustatory cortex neurons typically activated by sucrose itself (Saddoris, Holland, \& Gallagher, 2009), reflecting Powers et al. (2017), where a visual cue paired with tones elicited activation in brain regions sensitive to auditory stimulation. In addition, auditory cues 
previously paired with sucrose can elicit positive hedonic responses typically produced by exposure to sucrose itself (e.g. Holland, Lasseter, \& Agarwal, 2008). Moreover, if sucrose is devalued by pairing it with illness after the auditory cue to sucrose pairings, then the auditory cue will elicit negative hedonic responses representative of the now devalued sucrose and not positive responses that would have been produced during the pairing stage.

Not only does Pavlovian conditioning allow one cue to activate the sensory and hedonic properties of another, but stimuli indirectly activated through previously established associations can engage in new learning through mediated conditioning or sensory preconditioning. An example of mediated conditioning (e.g. Holland, 2005) involves initial Pavlovian conditioning to establish a tone-food association. The tone is then presented without the food and followed by the induction of illness. This results in the formation of an aversion to the food despite it never being directly paired with illness. Holland attributed this to the auditory cue activating perceptual processing of the food, and thus the percept of food was paired with illness despite the absence of food on the aversion learning trials. Taken together, these animal-lab results suggest that sensory and hedonic properties of stimuli are reactivated and experienced following presentation of cues with which they were previously paired. In short, it appears that rats, like humans, can experience a precept or hallucination of an absent stimulus when it is activated after associative learning.

While these animal-based studies imply convergence with humans in terms of the products of Pavlovian conditioning and its ability to evoke the perception of absent events, they also imply that there are boundaries to these effects. In particular, the effects produced by associative activation of an absent cue appear to depend on the amount of training. After short periods of training (16 tone-sucrose pairings) presentation of the tone alone elicited the same hedonic responses seen to sucrose itself, but no such effect was seen after long periods of training (112 tone - sucrose pairings) - despite that fact that other behavioural responses (such as food-magazine approach) were unaffected (Holland et al., 2008). Similarly, mediated conditioning was seen after short but not long training, despite there being no reduction in conditioned responding to the tone after extended training (Holland, 2005). It seems that limited amounts of Pavlovian conditioning allow CSs to evoke perceptual processing of their associated USs, but this ability is lost after extended training. This might contribute to the group differences reported by Powers et al. (2017) if participants prone to hallucinations are less susceptible to changes in the quality of the association over time. Although there is no direct evidence, Bayesian modelling did suggest that participants in the hallucination groups were less sensitive to degradation in the visual-auditory contingency across testing.

Considering the results of Powers et al. (2017), alongside the prior animal conditioning literature, suggests strong similarities between them. Perhaps the most intriguing possibility raised is that it suggests ways of investigating hallucination-like behaviour in the animal laboratory. If Pavlovian conditioning between a CS and a US allows subsequent presentation of the CS to elicit perceptual processing of the US akin to hallucinations in some circumstances, and the tendency for this to occur is greater in individuals prone to hallucinations, then observing a high degree of CS-elicited perceptual processing of the US could be an indication of high susceptibility to hallucinations. Moreover, the procedures considered above provide a number of alternatives for assessing the degree of US perceptual processing elicited by CSs. For example, sensory preconditioning relies on CS eliciting perceptual processing of the US, and so animals subject to associatively induced hallucinations should demonstrate strong mediated conditioning effects. Similarly, because the ability of a cue paired with sucrose to evoke hedonic responses appropriate to sucrose is determined by the cue eliciting sensory processing of sucrose, then animals subject to associatively induced hallucinations should demonstrate strong conditioned hedonic responses. These possibilities are particularly interesting in the context of animal models, because hallucinations are a central but little understood symptom of psychosis. And while there has been a concerted effort to develop principled methods for translational studies of cognition in schizophrenia (Moore, Geyer, Carter, \& Barch, 2013), there are currently no established behavioural measures for the study of hallucinations.

In summary, Powers et al. (2017) provide an elegant demonstration of associatively induced hallucinations and an insight into the contribution of top-down cognitive processes in perception to the susceptibility to hallucinations. The similarity between their results and prior studies in rodents suggests a continuity of process and experience between humans and other animals. Thus, not only have Powers et al. (2017) advanced the study of hallucinations in humans, but they point the way for additional translational research in laboratory animals.

\section{References}

Holland, P. C. (2005). Amount of training effects in representationmediated food aversion learning: No evidence of a role for associability changes. Learning \& Behavior, 33(4), 464-478.

Holland, P. C., Lasseter, H., \& Agarwal, I. (2008). Amount of training and cue-evoked taste-reactivity responding in reinforcer devaluation. Journal of Experimental Psychology-Animal Behavior Processes, 34(1), 119-132.

Moore, H., Geyer, M. A., Carter, C. S., \& Barch, D. M. (2013). Harnessing cognitive neuroscience to develop new treatments for improving cognition in schizophrenia: CNTRICS selected cognitive paradigms for animal models. Neuroscience and Biobehavioral Reviews, 37(9), 2087-2091.

Powers, A. R., Mathys, C., \& Corlett, P. R. (2017). Pavlovian conditioning-induced hallucinations result from overweighting of perceptual priors. Science, 357(6351), 596-600.

Saddoris, M. P., Holland, P. C., \& Gallagher, M. (2009). Associatively learned representations of taste outcomes activate taste-encoding neural ensembles in gustatory cortex. Journal of Neuroscience, 29(49), 15386-15396. 